\title{
On the empirical coherence and the spatiotemporal gap problem in quantum gravity: and why functionalism does not (have to) help
}

\author{
Niels Linnemann ${ }^{1}$ (D) \\ Received: 4 January 2019 / Accepted: 8 April 2020 / Published online: 6 May 2020 \\ (c) The Author(s) 2020
}

\begin{abstract}
The empirical coherence problem of quantum gravity is the worry that a theory which does not fundamentally contain local beables located in space and time-such as is arguably the case for certain approaches to quantum gravity-cannot be connected to measurements (which are actually carried out in space and time) and thus has its prospects of being empirically adequate undermined. Spacetime functionalism à la Lam and Wüthrich (Stud Hist Philos Mod Phys 64:39-51, 2018) is said to solve this empirical coherence problem as well as bridging a (putatively) severe conceptual gap between spatiotemporal structures of classical spacetime theories on the one hand, and the (so-called) non-spatiotemporal structures in quantum gravity approaches on the other hand (call this the spatiotemporal gap problem). The aim of this essay is to offer a deflationary account of both the empirical coherence problem and the spatiotemporal gap problem as they are claimed to arise at least prima facie for current theories of quantum gravity by Huggett and Wüthrich (Stud Hist Philos Mod Phys 44(3):276285, 2013), Lam and Wüthrich (2018) and Le Bihan (Synthese 2019. https://doi.org/ $10.1007 / \mathrm{s} 11229-019-02449-6)$. I defend the view that (1) spacetime functionalism is set up to address a problem (the empirical coherence problem) which can usually be solved without it; and that (2) it is wrongly claimed to (dis)solve another problem which for any actual account of quantum gravity is in fact currently non-existent anyway (the spatiotemporal gap problem).
\end{abstract}

Keywords Spacetime functionalism - Quantum gravity · Space · Time ·

Functionalism - General relativity · Empirical interpretation · Empirical coherence

Niels Linnemann

niels.linnemann@uni-bremen.de

1 Institute of Philosophy, Enrique-Schmidt-Str. 7, 28359 Bremen, Germany 


\section{Contents}

1 Introduction . . . . . . . . . . . . . . . . . . . . . . . 396

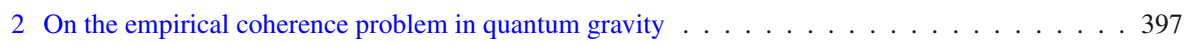

2.1 The misconceived empirical (in)coherence issue . . . . . . . . . . . . . . . . . . 398

2.2 Proper issues of empirical incoherence . . . . . . . . . . . . . . . . . . . . . . . . . . . . . . . . . . . . . . . . . .

3 On the spatiotemporal gap problem in quantum gravity . . . . . . . . . . . . . . . 405

3.1 Why there is no spatiotemporal gap problem in current approaches to quantum gravity . . . . 405

3.2 Why spacetime functionalism could not solve the spatiotemporal gap problem in any case . . 408

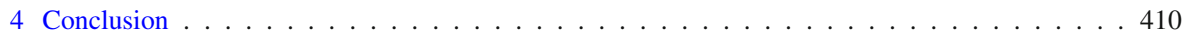

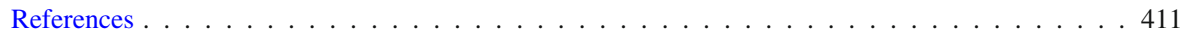

\section{Introduction}

The empirical coherence problem of quantum gravity is the worry that a theory which does not fundamentally contain local beables located in space and time-such as arguably the case for certain approaches to quantum gravity-cannot be connected to measurements (which are actually carried out in space and time) and thus has its prospects of being empirically adequate undermined. Spacetime functionalism à la Lam and Wüthrich (2018) is said to solve this empirical coherence problem as well as bridging a (putatively) severe conceptual gap between spatiotemporal structures of classical spacetime theories on the one hand, and the (so-called) non-spatiotemporal structures in quantum gravity approaches on the other hand (call this the spatiotemporal gap problem from hereon). ${ }^{1}$

Now, the aim of this work is to offer a deflationary account of both the empirical coherence problem articulated by Huggett and Wüthrich (2013) and the spatiotemporal gap problem for current theories of quantum gravity as it has been set up by Huggett and Wüthrich $(2013)^{2}$ and expanded on by Lam and Wüthrich (2018). (Lam and Wüthrich (2018) are also deflationary about the problem. But unlike them, I do not question its feasibility per se but rather that it occurs within our best candidates for a theory of quantum gravity.) Going beyond the current status quo in quantum gravity research, I then claim that functionalism could not solve anything genuinely deserving the name of a spatiotemporal gap problem anyway. Put a bit provocatively, I thus arrive at the view that (1) spacetime functionalism is set up to address a problem (the empirical coherence problem) which can be solved without it (namely in terms of what is known as empirical interpretation); and that (2) it is (wrongly) said to (dis)solve another

\footnotetext{
1 The spacetime functionalism of Lam and Wüthrich (2018) needs to be distinguished from that of Knox (2013), which is first and foremost concerned with what we mean by phenomenological spacetime structure within classical (so-called) spacetime theories. On Knox's account,
}

spacetime is whatever serves to define a structure of inertial frames, where inertial frames are those in whose coordinates the laws governing interactions [footnote suppressed] take a simple form (that is universal insofar as curvature may be ignored), and with respect to which free bodies move with constant velocity. (Knox 2017, p. 5)

2 See Sect. 3. 
problem which for any actual account of quantum gravity is in fact non-existent (the spatiotemporal gap problem). ${ }^{3}$

More precisely, I will proceed as follows: In the first part of the essay (Sect. 2), I address the empirical coherence issue and argue how it is better understood as an issue of empirical interpretation. The empirical coherence problem of spacetime emergence in quantum gravity is thereby unmasked as no more interesting from a philosophical point of view than the issue of empirical interpretation in other theories of modern physics. I will however point out that an interesting empirical coherence problem can prima facie arise at the level of individual models in quantum gravity approaches-as opposed to at the level of theories, that is for every model of a theory. ${ }^{4}$ But this is, as I argue, not novel in the context of models of 'spacetime' theories either. Conceiving of the empirical coherence problem as special to quantum gravity, thus in any case misses the point.

In the second part (Sect. 3.1) — getting to the spatiotemporal gap problem-I argue that the spatiotemporal gap problem needs to be fleshed out in the following way: any qualitative difference between something space(like) and time(like) disappears in the so-called non-spatiotemporal structure. As a split between something space(like) and something time(like) is still present in quantum gravity approaches (see Le Bihan and Linnemann 2018), the spatiotemporal gap problem does not occur in quantum gravity as we currently know it. If the spatiotemporal gap problem did occur, functionalism would not be able to explain it away anyway. (This is the topic of Sect. 3.2.)

\section{On the empirical coherence problem in quantum gravity}

Huggett and Wüthrich (2013) are interested in the empirical coherence of quantum gravity approaches. A theory is defined as empirically incoherent "in case the truth of the theory undermines our empirical justification for believing it to be true." (Huggett and Wüthrich 2013, p. 277). First, Huggett and Wüthrich (2013) make the assumption that empirical science ultimately rests on tracking pointer coincidences sorted by place and time stamps (known as 'beables'):

A central concern of philosophy of science is understanding how the theoretical connects to the empirical, the nature and significance of 'saving the phenomena'. ...At a high level of generality, however, presumably the link is established by observing (in some sense) a material 'something', in some determinate state

\footnotetext{
3 Lam and Wüthrich (2018), for instance, take their spacetime functionalism to dissolve the spatiotemporal gap problem as follows
}

[F]rom a functionalist point of view, nothing remains beyond showing how the fundamental degrees of freedom can collectively behave such that they appear spatiotemporal at macroscopic scales in all relevant and empirically testable ways. This turns out to be a hard task in quantum gravity. Functionalism can be seen as the assertion that once this task is completed, no unfinished business lingers on. (p. 44)

4 I am taking use of the semantic account of theories here, which renders theories as a set of models. See Winther (2016), in particular section 3.1.2, and references within. A locus classicus on the semantic account is Van Fraassen (1980). 
or other, at some spatial location at some moment in time and connecting this occurrence to our theory, for instance by postulating, in our theory, entities which behave in ways that would explain our observation. This is crude, no doubt, but seems to capture quite generally the nexus between our theorising about the world and our experiencing it, from meter readings in the lab to observing distant galaxies with a radio telescope to the results of high energy collisions. (p. 276)

Then, the general concern of Huggett and Wüthrich towards quantum gravity is that (many) approaches to quantum gravity (at least at first sight) feature empirical coherence issues, as their actual structures are so distinct from familiar spacetime theories that they either already do not allow for local beables at the fundamental level or at least do not suggest a clear path for connecting the familiar local beables at the derivative level (those beables we are using and referring to in our phenomenal spacetime) to the local beables in the fundamental theory. The issue resembles the debate on $3 N$-dimensional (configuration) space vs. 3-dimensional (position) space in the philosophy of (non-relativistic) quantum mechanics, and the specific question therein of how one can make sense of the idea that $3 N$-dimensional space could ground a $3 d$-dimensional space if indeed more fundamental. ${ }^{5}$ In both the QG and the QM case, the putative empirical coherence threat is thus rooted in the lack of fundamental beables and/or a lacking connection to observable beables.

In response to Huggett and Wüthrich's concerns with empirical coherence in quantum gravity approaches I will work through the following points:

- Strictly speaking, empirical coherence can only occur in models of an empirically relevant physical theory but not in a physical theory as such. Moreover, empirical coherence worries would be abundant in modern physics if they were just about the lack of local beables at the fundamental level of the theories (Sect. 2.1).

- An interesting example for a spacetime theory whose models partly feature empirical coherence issues is given by general relativity (GR). Thus, empirical coherence issues at the model level even among spacetime theories are not special to quantum gravity but already occur in GR (Sect. 2.2), rendering their occurence in approaches to $\mathrm{QG}$ as less dramatic.

\subsection{The misconceived empirical (in)coherence issue}

Theoretical laws can only be tested through testing the empirical laws derived from them. ${ }^{6}$ In this sense, it would be wrong to believe that an approach to quantum gravity (as a candidate for an empirical theory) itself can bring along an issue of empirical coherence; a genuine issue of empirical coherence could only arise if the laws of quantum gravity were not connected to empirical laws at all. This will however not be the case for any viable approach to quantum gravity, as it is required to reduce to GR or at least to a theory empirically equivalent to GR for the regime within which GR has

\footnotetext{
5 See for instance Ney and Albert (2013), and Ney (2017) for a discussion.

6 I am referring here to the usual distinction between theoretical and empirical laws based in turn on an (arguably not absolutely sharp) distinction between theoretical and empirical vocabulary (as familiar from logical empiricism). In short, empirical laws are expressed in terms of theoretical and empirical terms, while theoretical laws only contain theoretical terms.
} 
been successfully tested. So, unlike claimed by Huggett and Wüthrich, it is not relevant in this context to 'understand' how spacetime emerges, that is say in the context of loop quantum gravity (LQG) how space-time arises from spin networks. It only matters that spacetime does emerge since otherwise the approach to quantum gravity is not worth considering in the first place. The idea of a theory of quantum gravity fully detached from current physics (that is a theory not reducing to any physical theory at all currently in use, or at least not even numerically agreeing with one of them in some regime) is far from any reality.

In other words, physical theories cannot be empirically incoherent-or else they are simply not physical but just mathematical structure. (If a theory was completely empirically incoherent in the first place, it would be disconnected to all currently known physical theories as they are after all linked to the empirical to some degree.) Still, one could argue that the relevant question is about whether currently known quantum gravity approaches are just mathematics or whether they can reduce to measurable statements. But this is to be decided by the common derivational work as usually done in the physics and not in the philosophy department.

Now, if one were to look for empirical coherence issues, one should rather expect them in particular models of theories not in (empirically relevant) theories as such. In fact, single models of more fundamental theories might not all be connected to models of their derivative theories nor to directly empirically relevant laws (see next section). (For saying that a more fundamental theory reduces a putatively less fundamental theory it is after all sufficient that all models of the less fundamental theory are reduced by models from the more fundamental theory-but not vice versa.) It is those kinds of models which we should count as 'empirically incoherent'. 7,8 Thus, rather than talking about how a theory is empirically incoherent "... in case the truth of the theory undermines our empirical justification for believing it to be true ..." (Huggett and Wüthrich 2013, p. 277), we should be interested in the notion of a model being empirically incoherent in case the truth of the model undermines our empirical justification for believing it to be true.

It could be objected to my complaints above that Huggett and Wüthrich themselves already concede with respect to most approaches they consider that there is no empirical coherence issue at the theory level linked to them. And that the decisive question is thus rather whether the empirical coherence worry towards (some) approach to quantum gravity is a legitimate philosophical prima facie problem. ${ }^{9}$ However, as I will argue in the following, accepting an empirical coherence issue for QG approaches as a relevant prima facie problem draws on an overly naive realist picture which ignores

\footnotetext{
7 Models which do not admit an empirical interpretation could — in a strict sense - even be seen as a failure of the theory: At least, their finding should encourage looking for an explicit reformulation of the theory-a variation of the theory-in which the problematic sector of models is excluded. Thanks to an anonymous referee for pressing me on this point.

8 One could even say that, luckily, empirical interpretation is a model-specific affair: this allows for using model-specific features, such as the availability of $3+1$-split (in the case of certain GR-spacetimes) to heavily facilitate empirical understanding. Thanks to an anonymous referee for bringing this point up.

9 That it could arise - and, as a matter of fact, has arisen-on some personal-psychological level from hypothetically stipulating that issue in the first place, or from taking claims to this effect at face value, which are made by philosophers of physics, popularisers of physics, or physicists, is, of course, without doubt.
} 
as to how much theories are generally in need of interpretation —and thus arguably hardly attractive.

In a first step, it is worth stressing that (i) a part of the empirical coherence issue (Huggett and Wüthrich 2013) have in mind cannot count as a merely epistemological one but that it presupposes some commitment to scientific realism. A pragmatic physicist clearly need not come up with this worry: Rather, once she has managed to mathematically reduce results from a quantum gravity approach to GR she will just go out—or let others go out—and measure quantum-gravitational corrections to GR quantities. It is in this sense that it is essentially a realist's worry.

In a second step, it is important to (ii) acknowledge that the empirical coherence issue of Huggett and Wüthrich (2013) could have been posed in an analogous way in other cases just as well (in the context of thermodynamics for instance) but that it was not: After all, just as it is true that there might be no local beables (at least not in the standard sense) available at the fundamental level in quantum gravity, thermodynamics does not feature beables in a standard sense, either (it is primarily a theory about how different equilibrium states can be linked to one another). We can only 'embed' thermodynamic systems into spacetime regions in a highly crude manner:

- Time is only brought into the theory in a very minimal sense, namely as the posit that systems not in equilibrium approach equilibrium (while systems in equilibrium remain in equilibrium — see Brown and Uffink 2001). In particular, there is no sense of duration for time in thermodynamics.

- Space only features in the theory indirectly via notions such as pressure and volume.

In particular, as thermodynamics is duration-less it can strictly speaking not be linked to determinate spacetime regions (it is not all clear how long a process will take). Assignment to a determinate spacetime region only becomes possible from past experience (based on previous experience with specific systems, one learns that the approach to equilibrium occurs within a certain time-span), or from statistical mechanics (which allows for making more quantitative statements about the approach to equilibrium). Now, given that the (classical mechanistic) sense of beables as a precise marker of events in space and time is not available in thermodynamics either, the naive realist could equally stumble into an empirical incoherence issue when facing thermodynamics: after all, she could wonder how a theory of thermodynamics without duration could be empirically adequate if it was not straightforwardly relatable to our phenomenal spacetime.

That the naive realist as a matter of fact did not stumble here, might strike one as an important disanalogy between the case of thermodynamics, and the case of QG approaches. Well, that we are not aware of any bigger empirical coherence issue in the face of thermodynamics, lies in the fact that thermodynamics happened to be developped as a generalisation of how heat machines, engines, and all that work (that is, as a phenomenological theory). Only later thermodynamics got abstracted into a more universal framework (whose interpretation on its own then, as argued, is not immediate anymore). In other words, possible interpretations of thermodynamics and linkages to the world were part of its development from the very beginning. Then, given the contingency of such a generative story, a naive realist would have to 
recognise a prima facie empirical coherence worry towards thermodynamics-if not as one that actually unfolded in our world, then at least in some (nomically identical) world in which thermodynamics was arrived at via a less phenomenological route. Note, however, that if the theory of thermodynamics is not seen to have suffered from an empirical coherence issue in our world but only in some (nomically equivalent) other one, the naive realist should also expect that some specific approaches to QG she currently labels as prima facie empirically incoherent will most likely not suffer from a prima facie empirical coherence issue in some other (nomically identical) world; say, because in that world we had already adapted our way of talking about physics, and doing experiments beforehand in such a way that the interpretation of the structures of these approaches in question becomes immediate. Fair enough. But, as long as not just a prima facie worry contingent on quite specific albeit $Q G$-independent background knowledge is meant by the empirical coherence issue in QG (but rather a general prima facie worry about the nature of specific QG approaches and their in-principle linkability to the world-as I will assume in the following), a naive realist will have to regard both thermodynamics and approaches to QG as suffering from a (prima facie) empirical coherence issue. ${ }^{10}$

In sum then, the empirical coherence worry can thus be said to only arise with full force on us realists when we ignore the general knowledge on how much physical theories — such as thermodynamics and not just QM or QG — stand in need of interpretation anyway (that is, if we are straightforwardly naive realists). To stress: None of this is to say that I disagree with the answer of Huggett and Wüthrich (2013) to the worry, namely that it can be dismissed through acknowledging that physical salience can flow from the derivative level to the fundamental level as well. My point is rather that there is no need to present the (so-called) empirical coherence problem as a genuine worry which could perhaps not be overcome: it is simply a task to address (a task of interpretation) albeit none whose eventual success is seriously at issue.

More precisely, and to put a positive spin on things, Huggett and Wüthrich (2013) (and also Lam and Wüthrich 2018) should thus simply be understood as making explicit (in particular to the naive realist) the specific interpretational need(s) linked to different approaches to quantum gravity. Generally, empirical interpretation is loosely about how to link the theory to the world. Curiel (2009) for instance introduces the notion of concrete interpretation:

Concrete. The fixation of a semantics for the formalism, in the sense that the formalism under the semantics expresses the empirical knowledge the framework contains - for example, the fixation of a Tarskian family of models, or, less formally, the contents of a good, comprehensive text-book. (p. 46)

\footnotetext{
10 Relatedly, one might make out a disanalogy between QG approaches and thermodynamics as that with respect to the latter but not with respect to the former we have (1) experimental access to the theory-specific regimes, and (2) a readily available, straightforwardly empirically relevant underlying theory. Now, both (1) and (2) are aspects at most only indirectly related to the question how the formal nature of the theory itself relates to the (measurable) world-again, the core of the empirical coherence issue about QG approaches. Therefore, it strikes me as if a disanalogy about (1) and (2) cannot be a decisive issue for the parallel drawn between QG and thermodynamics. Thanks to an anonymous reviewer for pressing me on this point.
} 
Every physical theory - at least with respect to some of its models—needs to have a concrete interpretation. Building on a further notion by Curiel (called 'metalinguistic interpretation'), a theory can be said to stand in need of empirical interpretation in an interesting sense if it either (1) can only be empirically understood upon providing sophisticated qualifications as to in what sense its empirical attributions apply, or (2) requires extratheoretical import to allow for or make sense of empirical attributions. Quantum mechanics proper is a prime example for a theory in need of interpretation in an interesting sense - a naive concrete interpretation of the formalism alone leaves it open how determinate measurement results are obtained. In contrast to this, empirical coherence is about whether a theory/model can be interpreted at all.

The need of quantum gravity for an empirical interpretation in an interesting sense is two-fold then:

- Any theory of quantum gravity needs an interpretation qua being a quantum theory. ${ }^{11}$

- Even when ignoring quantum mechanical superposition effects, a theory of quantum gravity needs an interpretation if it rests on spacetime-less structures which do not allow for straightforwardly connecting to our usual 'beables'/familiar measurement procedures in $4=3+1$ dimensional spacetime. For instance, borrowing an example from Huggett and Wüthrich (2013), target space with respect to which string theory is standardly formulated only superficially looks like a higherdimensional generalisation of $4 \mathrm{~d}$ dimensional (curved) spacetime. Provided their assessment is correct, string theory itself is only empirically understandable via approximation and limit procedures since we can only this way make contact to expressions associable to objects in $4 \mathrm{~d}$ spacetime. A similar observation could be made with respect to the spin-network structures of LQG, causal sets in causal set theory, and so on (for details see the tour de force on what is left of spacetime in various approaches to quantum gravity by Huggett and Wüthrich 2013).

\subsection{Proper issues of empirical incoherence}

In the previous section, it was argued that the notion of empirical coherence in quantum gravity should be considered only at the level of single models but not at the level of theories, i.e. whole sets of models.

For example: Although LQG in so far as it is an approach to quantum gravity is empirically coherent, some LQG models will not lead to GR spacetime models (or any spacetime-like models) upon applying the appropriate reduction scheme (see Wüthrich 2006, p. 169). However, as I will explain in this section now, already our currently best spacetime theory-GR-features an analogous empirical coherence issue at the level of its models. (In other words, there is nothing special about the existence of an empirical coherence issue to quantum gravity qua being a spacetime theory either.)

Empirical access in GR ultimately rests on the availability of a coordination procedure, that is a protocol for how a relativistic observer can set up spacetime coordinate frames in order to sort and keep track of measurement results (one might very well

\footnotetext{
11 This presupposes of course that a theory of quantum gravity does not (sans interpretation or supplementation) solve the measurement problem — and is 'quantum' to begin with.
} 
in the end use a coordination scheme without an operational meaning for calculations etc. but this is a different matter). I will now argue that certain models do not even allow for such a coordination, and must thus count as empirically incoherent. ${ }^{12,13}$

Now, the only fully operationalist protocol for setting up (local) coordinates in a general relativistic spacetime ${ }^{14}$ employs radar (light) signals: light signals and their echos are used to probe an observer's environment relative to an (admittedly) arbitrary parameterisation of her own worldline $C_{0}: t \mapsto \gamma(t)$ (which serves as a time standard). In particular, the observer does not need to have a standard clock at her disposal, that is a clock that would show a reading proportional to the proper time of the observer. ${ }^{15}$ For a sent-off time $t_{1}$ and return time $t_{2}$ the following (local) radar coordinates $^{16}$ are then assigned to an event $P$ relative to the observer's worldline parameterisation $t \mapsto \gamma(t)$ (see also Fig. 1): ${ }^{17,18}$

$$
\begin{aligned}
T & =\frac{1}{2} \cdot\left(t_{1}+t_{2}\right) \\
R & =\frac{1}{2} \cdot\left(t_{2}-t_{1}\right)
\end{aligned}
$$

A direction with respect to which the signal is emitted needs to be determined relative to a (infinitesimally close-by) neighbouring worldline $C^{\prime}$. We can then say that two signals are emitted in the same direction if both cross this same neighbouring worldline. ${ }^{19}$ How the direction is exactly picked, is not important for the following, however. ${ }^{20}$

\footnotetext{
12 Thanks to James Read for in-depth discussions on the topic. Some of this will be the topic of a more comprehensive joint work on the epistemology of spacetime.

13 An argument similar in spirit is made by Brown (2005, p. 101) in his critique of Malament's (1977) putative resolution of the conventionality problem in special relativity; Brown shows how the notion of spacetime adhered to is operationally empty and thus un-physical. Thanks to an anonymous reviewer for pointing this out to me.

${ }^{14}$ See Perlick (2008), and Synge (1960), in particular pp. 76-102.

15 This renders the need for clocks in any more narrow sense unnecessary—contra (Synge 1960).

16 To avoid confusion note (single-observer) radar coordinates à la (Perlick 2008), and not (two-observer) radar coordinates à la (Ehlers et al. 2012) are used.

17 Arguably, one can choose $T=p t_{1}+(1-p) t_{2}$ for any $p \in(0,1)$, more generally. Note though that only for the choice of $p=1 / 2$ the coordinate chart can be smoothly continued at $R=0$. See Perlick (2008) and references within for a discussion; the locus classicus for this (putative) conventionality issue is Reichenbach (2003), §19.

18 In the case that the observer's wordline is parameterised by proper time, as measured by an ideal clock, one talks of proper radar coordinates. See for instance Minguzzi (2005).

19 Synge (1960) nicely explains the intuition behind this:
}

This is what an astronomer does in observing a star, $C_{0}$ being the world-line of his eye and $C^{\prime}$ that of the middle point of the object glass of his telescope. (p. 118)

20 Among other things, a bouncing photon experiment in which photons are sent off and caught again can for instance be used to pick a neighbouring worldline as a directionality reference (and thus a spatial reference frame) such that the frame is non-rotating. See Synge (1960, Ch. III, §6). 
Fig. 1 Radar coordinates for an event $P$ relative to observer on worldline $\gamma$

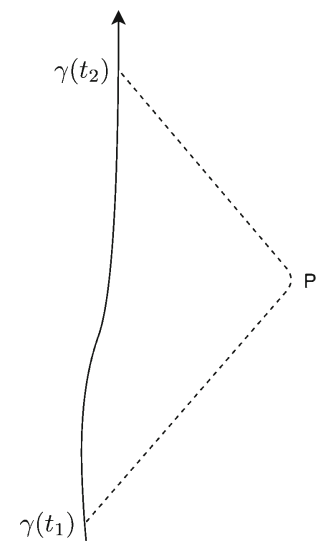

Provided that light signalling is available in the first place, radar coordinates are guaranteed to exist within a sufficiently small neighbourhood of any GR spacetime (see Proposition 1 in Perlick 2008). But unlike often assumed (say in the constructive axiomatic approach to GR of Ehlers et al. 2012), light signals simply do not necessarily belong to the idealised ontology of GR. After all, light is internally first of all described by the electrodynamics sector of GR, and can as such only be said to trace out null geodesics in an idealised fashion if what is known as the geodesic-optic limit holds (that is if light wave packets can indeed be shown to be idealised as rays tracing out null geodesics when taking a high frequency limit). ${ }^{21}$

Following Asenjo and Hojman (2017), light signals will in fact not follow null geodesics in Gödel spacetime (neither in rotating spacetimes more generally) but move at varying speed. ${ }^{22}$ As a consequence, at least in Gödel spacetime, radar coordinates cannot be set up since light signals will not generally move on null geodesics and can thus not be used as a fixed, coordinate-independent reference standard (thus, suffering from the same problems like timelike signals). However, given that radar coordinates (or radar-type coordinates) are the only notion of operational coordination available, sorting and recording events thus becomes-even under all forms of idealisationimpossible. $^{23}$

21 Likewise, test particles can only be seen as an idealised ontology for GR if this is compatible with the matter sector assumed. See, for instance, Tamir (2012), in particular Sect. 4 on limit operations proofs for the geodesic principle (such as that of Geroch-Jang) which build on specific assumptions on the energymomentum tensor.

22 More precisely, Asenjo and Hojman (2017) demonstrated that the group and phase velocity of general electromagnetic waves are not constant in Gödel spacetime, and that the geodesic-optic limit can thus not be taken. It can be shown that the signalling speed linked to light — the front velocity - is equally varying if the concept of a wave front is at all still applicable (so either signal propagation by light happens at varying speed then, or is not possible at all). See Menon and Linnemann (2018).

23 One might think that, even if one was able to operationally set up and take use of a reference frame in Gödel spacetime_-despite the lack of a reliable signalling method—, the full metric could not be measured out in any case: after all, no objects would be available to accurately trace out the conformal structure (that is the structure of null geodesics); but knowledge of this structure is required for the full reconstruction of the metric on the standard constructive axiomatic accounts of GR (see Ehlers et al. 2012, and also Korté 182). Note though that-in order to determine the metric field—one need not proceed by directly tracking 
We do not need to go into detail here. What matters for us, is that we have found that the very basis for empirical access in GR — radar(-like) coordinates-cannot (not even in an idealised fashion) be set-up in certain spacetime models (first and foremost not in Gödel spacetime). In a lack of an operationalist sense of coordinates, pointer coincidences cannot be kept track of and in this sense there are effectively no local beables to work with. Models like Gödel spacetime are thus first of all empirically incoherent. This is not to exclude that there might be some operational procedure from outside of GR - say quantum information - for circumventing the sketched obstacles to setting up radar coordinates, and tracking point coincidences but, from a GR point of view, the mentioned spacetimes are just empirically incoherent. ${ }^{24}$

\section{On the spatiotemporal gap problem in quantum gravity}

We so far addressed the empirical coherence problem in quantum gravity: The empirical coherence problem of spacetime emergence in quantum gravity was revealed to be no more interesting from a philosophical point of view than issues of empirical interpretation in other theories of modern physics. We saw that an interesting empirical coherence problem - again, not exclusive to quantum gravity-could only arise at the level of individual models of certain quantum gravity approaches. In the next two sections, we will dedicate ourselves to the spatiotemporal gap problem. I first argue that there is nothing in the relationship between current quantum gravity approaches and GR calling for the label of a specific spatiotemporal gap problem. I then go on to argue why functionalism could not even solve a genuine spatiotemporal gap problem if it did occur in the context of a theory of quantum gravity.

\subsection{Why there is no spatiotemporal gap problem in current approaches to quantum gravity}

The spatiotemporal gap problem is a specific conceptual gap problem between a putatively non-spatiotemporal theory and a derivative spatiotemporal theory, first and foremost set up by Le Bihan (2018a,b) and Le Bihan and Linnemann (2018), and further illustrated in Le Bihan (2019). To be clear: surely, intertheory relations generically involve conceptual gaps. But the issue here is with the specific conceptual issue of how the non-spatiotemporal theory can lack essential features of the spatiotemporal but still ground the spatiotemporal theory.

null and timelike geodesic structure (known as the causal-inertial method, see also Bell and Korté 2016, $\S 4.3$ for a helpful introduction). Rather, one could equally track the structure of all timelike curves, and that of timelike geodesics specifically (as knowing all timelike curves will allow for knowing the null geodesic structure as well, see Malament 1977).

24 There is a slight disanalogy between the QG case and the GR case insofar as that 'having no beables at the fundamental level' (as the case for certain QG approaches) is distinct from 'not being able to operationally take use of beables at the fundamental level' (as the case for some GR models). Nevertheless whether local beables do not exist or whether they are just not in principle operationally accessible — both amounts to an empirical coherence issue rooted in the inaccessibility of the fundamental beables (if beables do not exist, they cannot be accessed either). 
Le Bihan (2019) makes an analogy to the qualia problem of consciousness: The spatiotemporal gap problem refers to an explanatory gap between non-spatiotemporal and spatiotemporal structures similar to the explanatory gap between physical states and the supposedly qualitative marks of mental states called qualia (such as what it is like to experience 'redness'). In reference to a well-known nomenclature by Chalmers (2011) who calls the issue of explaining qualia from physical states the hard problem of consciousness, Le Bihan then dubs the problem of bridging the conceptual gap between the non-spatiotemporal and the spatiotemporal the hard problem of spacetime emergence. No matter how well this analogy fares as such, ${ }^{25}$ the choice to call the spatiotemporal gap problem a hard problem makes clear that it is not at all meant as yet another conceptual gap problem as it generically occurs in theory change, including theory change between spacetime theories.

Now, Le Bihan 2019 considers in detail which forms of spacetime functionalism solves or dissolves the problem (i.e., renders the problem moot). Lam and Wüthrich (2018), for instance, take their spacetime functionalism to dissolve the hard problem. ${ }^{26}$ In contrast, the question I am going to ask now is what a genuine spatiotemporal gap problem - of a comparable severity of the hard problem from consciousness - has to do with quantum gravity as we currently know of it from our best approaches in the first place (spoiler: not much).

First, note that the occurence of the problem clearly depends on what we take to be the criteria of the spatiotemporal. We can set the bars for what to call spatiotemporal high, or low - that is try to look for a sense of the spacetime close to our own rich phenomenological understanding of the spatiotemporal or rather opt for some minimal sense of the spatiotemporal. Depending on how we define spatiotemporal, we thus already have had several changes from the non-spatiotemporal to the spatiotemporal. Take the following examples of theory changes:

- Among other things, the theory change from SR to Newtonian mechanics gets from a notion of the spatiotemporal without absolute simultaneity to one with absolute simultaneity.

- Among other things, the theory change from GR to SR gets from a notion of the spatiotemporal without a global time direction to one with a global time direction.

But surely, none of this seems to be a deep conceptual gap as meant by the spatiotemporal gap problem: after all, there are many features about space and time we are more or less easily willing to give up, and which we do not need to presuppose. This does of course not change when facing quantum gravity: many features different from GR spacetime like discreteness, disordered locality, ${ }^{27}$ and superposition of metric structure we find in quantum gravity do not straightforwardly mean that these approaches are non-spatiotemporal. Most marks of the spatiotemporal brought up explicitly are either easily acceptable as contingent (such as discreteness) or external (at least) to physics (such as tense- that is to say that these conceptual marks cannot be captured

\footnotetext{
25 For an apt defense, see Le Bihan 2019.

26 See footnote 3.

27 As pointed out by Huggett and Wüthrich (2013), some relations of adjacency in LQG's spin networks map onto large distances at the low-energy, classical level. Thereby, the sense of locality at high-energies does not map onto the sense of locality at low-energies.
} 
by our physics (see Dolev 2018) and arguably not by our senses either (see Frischhut 2015)).

We could continue to argue about what's spatiotemporal and what's not, clinging to our background intuitions. But then, whether the hard problem occurs is simply a subjective question (maybe it already occurs then with the change from SR to Newtonian mechanics). A serious spatiotemporal gap problem should be sufficiently robust under disagreement about which particular feature we take to be the mark of the spatiotemporal and which not. A clear spatiotemporal gap problem thus builds on the most minimal spatiotemporal features everyone would agree on. ${ }^{28}$ I claim these features are that of being some kind of ordering structure and involving some kind of difference between something timelike and something spacelike (these are the minimal marks of the spatiotemporal). Add and remove to our current conception of space and time whatever you want (as is arguably the case for several approaches to quantum gravity which draw a picture of geometric deviation from classical spatial structure, ${ }^{29}$ temporal discreteness in contrast to classical temporal continuity, and even quantum mechanical superposition of space and time) but the only feature that you apparently cannot do away with through any of these approaches is the idea that space and time are locally in some sense distinct, and together serve as ordering structure! So, only if the so-called non-spatiotemporal was not even minimally spatiotemporal in the above sense would there be a clear and non-disputable gap between the non-spatiotemporal and the spatiotemporal radically different from the gaps we already know from SR to Newtonian mechanics or GR to SR.

But now it simply turns out that there is still a minimal sense of spatiotemporal structures in current QG approaches. More precisely, looking at our currently best guesses on what could end up as a theory of quantum gravity, we simply do find that the split of time and space familiar from our currently best physics ${ }^{30}$ persists in one form or another (unlike any other feature typically linked to spacetime). This has been argued for in more detail in Le Bihan and Linnemann (2018): Each approach to quantum gravity encodes a split between something (quasi-)spatial and something (quasi-)temporal; for instance, through a representation of the Lorentz group (such as in perturbative GR, perturbative string theory, asymptotic safety, covariant LQG), by partial order (causal set theory), or by foliation prior to quantisation (canonical quantum gravity, canonical LQG). ${ }^{31}$ (Without doubt, the methodology at play here is naturalist in nature-it comprehensively takes our current and putatively best future physical theories seriously-and therefore of course in principle becomes subject to

\footnotetext{
28 Cf. Baker (2019) who has argued that the notion of 'spacetime' is best understood across physical theories as a cluster concept, that is "a concept with multiple criteria of application, none of which are necessary conditions for a thing to fall under the concept" (p. 15). Hereby, Baker, explicitly rejects that the concept of spacetime "has a primitive essence" (p. 19). The view argued for in this paper is compatible, however, with a restricted cluster concept view on spacetime on which the notion of spacetime is at some level of detail only conceivable as a cluster concept while it can at a sufficiently high level of abstraction still be constrained through necessary criteria.

29 As it arguably occurs for LQG, see Huggett and Wüthrich (2013).

30 See Callender (2017), in particular chapter 6.

31 See Le Bihan and Linnemann (2018), table 1.
} 
refutation; this can, however, be seen as a strength rather than a weakness of the employed methodology.)

Finally, it is worth stressing that none of this is to say that theories of quantum gravity are not interesting conceptually in how they differ from GR spacetime etc. The point is only that there is no in principle different conceptual gap to deal with than we have been dealing with when, for instance, going from pre-relativistic to relativistic theories.

\subsection{Why spacetime functionalism could not solve the spatiotemporal gap problem in any case}

As noted before, the spatiotemporal gap problem does not occur within current quantum gravity approaches. Let's nevertheless assume for a moment that the spatiotemporal gap problem would show up: ${ }^{32}$ As we noted before, the two minimal marks of the spatiotemporal are that it provides ordering structure, and that there is a (local) split between something time(like) and something space(like). That the spatiotemporal is an ordering structure is arguably a necessary requirement that, locally, a difference between some time(like) and some space(like) directions can be set up. Generally speaking, without any form of basic ordering structure it seems as if no physical structure can be set up in the first place. I will thus focus on the apparently more interesting question of how far a local split between something time(like) and something space(like) can appear out of an ordering structure without it. In the following I will argue that functionalism would not provide the right toolkit for dealing with this issue.

Before we consider whether functionalism would solve the spatiotemporal gap problem, let us remind ourselves that functionalism cannot straightforwardly address the spatiotemporal gap problem of naturalising qualia in the philosophy of mind. As Le Bihan (2019) acknowledges:

Functionalism, in the philosophy of mind, aims at solving the mind-body problem by functionalising mental states, namely identifying them with functional roles realised by physical states. Functionalism aims at explaining the (or some) specific marks of the mental with respect to physical entities, namely intentionality and phenomenal consciousness. As I mentioned above, the strategy is regarded as successful when it comes to the intentional aspect of mental states. However, the predominant view in the philosophy of mind is that we may not functionalise qualia and physicalists rather aim at removing qualia from our ontological picture of the world, for instance through the phenomenal concepts strategy (Loar 1990), or by subscribing to illusionism (Frankish 2016). (p. 15)

This of course — as Le Bihan (2019) rightly points out—raises the immediate question: why take functionalist accounts to solve the spatiotemporal gap problem in

\footnotetext{
32 Or at least appear to show up. After all, spacetime functionalism is depicted by its main proponents as dissolving the hard problem, i.e. revealed as an illusion in the first place (see footnote 3 , but otherwise also the detailed discussion by Le Bihan 2019 on this matter). In this case, read 'solve' in the following as 'dissolve'.
} 
spacetime if they cannot solve the qualia problem in the philosophy of mind? In fact, in my opinion the task the functionalist has to solve in addressing the spatiotemporal gap problem of spacetime emergence is even more daunting than the (arguably) analogous one in the philosophy of mind: Spacetime functionalism faces a devastating dilemma.

On the one hand, spacetime functionalism could take its own metaphorical slogan 'spacetime is as spacetime does' more or less literally. But then the slogan has to build on diachronicity: that there is change first and foremost over time/over something timelike-rather than over space/over something spacelike. A minimum sense of diachronicity now requires that there is a local split between something time(like) and something space(like). The existence of the split in turn is, however, what one would like to explain away in the first place. On the other other hand, the slogan could explicitly deny requiring diachronicity. But then it amounts to nothing more than a verbalisation of what is already in the physics. I will expand on both horns of the dilemma in the following.

Start with two examples: (1) A water pump is whatever can get water from an (at least initially) energetically favorable reservoir into another reservoir. Things/structures/setups/... fulfill the role of a water pump as long as they do the pumping over time. This must be contrasted to (2) a mathematical function $f: \mathbb{R} \rightarrow \mathbb{R}, x \mapsto x^{2}$ which maps (in a well-defined manner) elements from one domain into another independently of time. ${ }^{33,34}$

On the one hand, saying that 'being spacetime' is a function (in a functionalist account) can be understood as that 'being spacetime' is a role played over time or something timelike just like 'being a water pump' is the role played over time of moving water from one reservoir into an (energetically-less favoured) other reservoir. This is the first horn of the dilemma. It is in this sense that the spacetime functionalist to GR à la Knox means that 'being chronogeometrically significant' or displaying a specific inertial structure is a role played by the $g$ field. ${ }^{35}$ In solving the hard problem of spacetime qualia, it is this required diachronicity we want to explain away however.

On the other hand, one might suggest that 'spacetime is as spacetime does' can at most be meant as a mere slogan for spacetime functionalism, and that the functionalist notions of 'doing'/'role'/... should not be taken to suggest diachronicity (at least not by necessity). ${ }^{36}$ This is the second horn of the dilemma. Spacetime functionalism along these lines (as for instance adhered to by Lam and Wüthrich (2018))

\footnotetext{
33 The notion of a computational function lies in-between these two, as a computational function can both be understood as a mere mathematical function and as a process over time (say when formalised as the process in a concrete instantiation of the Turing machine; including calculation of its halting time).

34 Temperature for instance should be seen as functionally reduced to particle movement in this mathematical sense. No one would say: particles play the role of 'temperature' though. The movement of particles rather simply instantiates a specific temperature.

35 See Knox (2017), p. 3: "the metric field is spacetime because of what it does [...] and not by way of what it is".

36 'Spacetime is as spacetime does' becomes incomprehensible as a metaphor then as well: a metaphor can only make sense if it is understandable in some literal fashion, and to make sense (literally) the metaphor of 'doing' and 'playing a role' presupposes that there is a remaining sense of (at least local) diachronicity left. This of course raises the question of why for instance (Lam and Wüthrich 2018) — who explicitly do not presuppose diachronicity in their spacetime functionalism — use the slogan in the first place.
} 
is now equally unsatisfactory since it amounts to nothing else than the claim that 'spacetime can be reduced from many different structures', the claim that GR spacetime is multiple-realisable from different underlying theoretical structures within one approach of quantum gravity. This in fact would however just be a (philosophicallyflavoured) reformulation of the claim that GR models are classical, low-energy limits of different models within one approach to quantum gravity, as this limit among other things involves coarse-graining. How such a qualification can in any way settle (an allegedly) deep conceptual issue of how spacetime comes out of less spatiotemporal structure - the hard problem (if there is one)—, is not clear: whether a time-split is multiple-realisable or not from a putative non-spatiotemporal structure, would not explain its occurrence.

\section{Conclusion}

The goal of this essay consisted in deflating the discussions around both the empirical coherence and the spatiotemporal gap problem in quantum gravity-including the alleged (dis)solution of the latter through a functionalist account (see Lam and Wüthrich 2018).

In the first part of the essay I argued that the empirical coherence problem of spacetime emergence is not peculiar to quantum gravity (or QM for what it's worth) but as such generic. It must be claimed to occur for thermodynamics as well, and surely_if it applies to QM-to QFT as well (and thus the whole Standard Model). The empirical coherence issue thereby becomes a prima facie threat to nearly all modern physical theories! As such then, it should better be understood as a problem of empirical interpretation. An interesting problem of empirical coherence should be rather looked for at the model-level but then again already spacetime theories prior to QG (like GR) feature such problems.

I then showed that there is no spatiotemporal gap problem occurring in the context of a current theory of quantum gravity, as what I took to be the main essential conceptual feature of space and time, that is the split between space and time, still occurs in any candidate theory to quantum gravity. Finally, I argued that functionalism does not have the resources to explain away a supposed spatiotemporal gap problem as it itself either (1) needs to assume a diachronic principle, and thereby implement a local split between something space(like) and time(like), or (2) just re-expresses the multiple-realisability of GR spacetime from underlying micro-structure - a point however orthogonal to the actual conceptual problem. Thereby, it was shown that (spacetime) functionalism could not solve the spatiotemporal gap problem even if there was one.

Two final remarks are in order to put the findings into context: (1) Clearly, it is too early to say that there cannot be a spatiotemporal gap problem-the appearance of a time-space split out of nowhere so-to-speak would count as one. The claim is only that there is none in the current approaches to quantum gravity (as all of them allow for identifying space-time splits at least within regions of their models). (2) Neither is any of this to suggest that the spatiotemporal gap problem could not be solved at all. However, the solution would not run through a (mere) functionalist approach: perhaps some sort of symmetry breaking across energy scales (in lack of a better 
analogy), ${ }^{37}$ leading to a split between (quasi-)space and (quasi-)time at lower energy scales would do the trick. But without any sensible model or physical intuition in this direction, the question whether such a solution was possible remains just as open as whether functionally realised mental states (such as in a computer) are sufficient for implementing the occurrence of qualia.

Acknowledgements Open Access funding provided by Projekt DEAL. I would like to thank Karen Crowther, Nick Huggett, Rasmus Jaksland, Baptiste Le Bihan, and Christian Wüthrich for extremely patient discussions and highly important feedback on the manuscript; and James Read for substantial discussions on chronometry and radar coordinates in general relativity specifically. I would also like to thank Richard Dawid and Fay Dowker for discussions on topics of the paper. Moreover, I am grateful to altruistic anonymous reviewers for helping me to substantially improve the manuscript. The project was made possible through the generous financial support by the Swiss Science Foundations (SNF) (Project No. 105212 165702).

Open Access This article is licensed under a Creative Commons Attribution 4.0 International License, which permits use, sharing, adaptation, distribution and reproduction in any medium or format, as long as you give appropriate credit to the original author(s) and the source, provide a link to the Creative Commons licence, and indicate if changes were made. The images or other third party material in this article are included in the article's Creative Commons licence, unless indicated otherwise in a credit line to the material. If material is not included in the article's Creative Commons licence and your intended use is not permitted by statutory regulation or exceeds the permitted use, you will need to obtain permission directly from the copyright holder. To view a copy of this licence, visit http://creativecommons.org/licenses/by/4.0/.

\section{References}

Asenjo, F. A., \& Hojman, S. A. (2017). Do electromagnetic waves always propagate along null geodesics? Classical and Quantum Gravity, 34(20), 205011.

Baker, D. J. (2019). On spacetime functionalism. http://philsci-archive.pitt.edu/15860/. Accessed 1 April 2020.

Bell, J. L., \& Korté, H. (2016). Hermann Weyl. In E. N. Zalta (Ed.), The Stanford encyclopedia of philosophy (winter 2016 ed.). Stanford: Metaphysics Research, Lab Stanford University.

Brown, H. R. (2005). Physical relativity: Space-time structure from a dynamical perspective. Oxford: Oxford University Press.

Brown, H. R., \& Uffink, J. (2001). The origins of time-asymmetry in thermodynamics: The minus first law. Studies in History and Philosophy of Science Part B: Studies in History and Philosophy of Modern Physics, 32(4), 525-538. https://doi.org/10.1016/S1355-2198(01)00021-1.

Callender, C. (2017). What makes time special?. Oxford: Oxford University Press.

Chalmers, A. (2011). Drawing philosophical lessons from Perrin's experiments on Brownian motion: A response to van Fraassen. The British Journal for the Philosophy of Science, 62(4), 711-732.

Curiel, E. (2009). General relativity needs no interpretation. Philosophy of Science, 76(1), 44-72.

Dolev, Y. (2018). Physics' silence on time. European Journal for Philosophy of Science, 8(3), 455-469. https://doi.org/10.1007/s13194-017-0195-z.

Ehlers, J., Pirani, F. A. E., \& Schild, A. (2012). Republication of: The geometry of free fall and light propagation. General Relativity and Gravitation, 44(6), 1587-1609.

Frischhut, A. M. (2015). What experience cannot teach us about time. Topoi, 34(1), 143-155. https://doi. org/10.1007/s11245-013-9211-x.

Huggett, N., \& Wüthrich, C. (2013). Emergent spacetime and empirical (in) coherence. Studies in History and Philosophy of Modern Physics, 44(3), 276-285.

\footnotetext{
37 Symmetry breaking across energy scales (certain symmetries only hold at low energies but not a high ones, or vice versa - as for instance the case in the grand unification scenario where certain symmetries among the fundamental interactions just show up from a certain energy scale on) should be distinguished from dynamical symmetry breaking (as in the Higgs mechanism).
} 
Knox, E. (2013). Effective spacetime geometry. Studies in History and Philosophy of Modern Physics, 44(3), 346-356.

Knox, E. (2017). Physical relativity from a functionalist perspective. Studies in History and Philosophy of Science Part B: Studies in History and Philosophy of Modern Physics,. https://doi.org/10.1016/j. shpsb.2017.09.008. Accessed 1 April 2020.

Korté, H. (1982). A realist interpretation of the causal-inertial structure of spacetime. Dissertation. https:// ir.lib.uwo.ca/digitizedtheses/1175. Accessed 1 April 2020.

Lam, V., \& Wüthrich, C. (2018). Spacetime is as spacetime does. Studies in History and Philosophy of Science Part B: Studies in History and Philosophy of Modern Physics, 64, 39-51. https://doi.org/10. 1016/j.shpsb.2018.04.003.

Le Bihan, B. (2018a). Priority monism beyond spacetime. Metaphysica, 19(1), 95-111.

Le Bihan, B. (2018b). Space emergence in contemporary physics: Why we do not need fundamentality, layers of reality and emergence. Disputatio. https://doi.org/10.2478/disp-2018-0004.

Le Bihan, B. (2019). Spacetime emergence in quantum gravity: Functionalism and the hard problem. Synthese. https://doi.org/10.1007/s11229-019-02449-6.

Le Bihan, B., \& Linnemann, N. (2018). Have we lost spacetime on the way? Narrowing the gap between general relativity and quantum gravity. Studies in History and Philosophy of Science Part B: Studies in History and Philosophy of Modern Physics,. https://doi.org/10.1016/j.shpsb.2018.10.010.

Malament, D. B. (1977). The class of continuous timelike curves determines the topology of spacetime. Journal of Mathematical Physics, 18(7), 1399-1404.

Menon, T., Linnemann, N., \& Read, J. (2018). Clocks and chronogeometry: Rotating spacetimes and the relativistic null hypothesis. The British Journal for the Philosophy of Science, , https://doi.org/10.1093/ bjps/axy055.

Minguzzi, E. (2005). The Minkowski metric in non-inertial observer radar coordinates. American Journal of Physics, 73(12), 1117-1121.

Ney, A. (2017). Finding the world in the wave function: Some strategies for solving the macro-object problem. Synthese. https://doi.org/10.1007/s11229-017-1349-4.

Ney, A., \& Albert, D. Z. (2013). The wave function: Essays on the metaphysics of quantum mechanics. Oxford: Oxford University Press.

Perlick, V. (2008). On the radar method in general-relativistic spacetimes. In H. Dittus, C. Lammerzahl, \& S. G. Turyshev (Eds.), Lasers, clocks and drag-free control. Astrophysics and space science library (Vol. 349, pp. 131-152). Berlin: Springer.

Reichenbach, H. (2003). The philosophy of space and time. Mineola: Dover.

Synge, J. L. (1960). Relativity: The general theory. Amsterdam: North-Holland Publishing Company.

Tamir, M. (2012). Proving the principle: Taking geodesic dynamics too seriously in Einstein's theory. Studies in History and Philosophy of Science Part B: Studies in History and Philosophy of Modern Physics, 43(2), 137-154.

Van Fraassen, B. C. (1980). The scientific image. Oxford: Oxford University Press.

Winther, R. G. (2016). The structure of scientific theories. In E. N. Zalta (Ed.), The Stanford encyclopedia of philosophy (winter 2016 ed.). Stanford: Metaphysics Research Lab Stanford University.

Wüthrich, C. (2006). Approaching the Planck scale from a generally relativistic point of view: A philosophical appraisal of loop quantum gravity. Ph.D. thesis, University of Pittsburgh.

Publisher's Note Springer Nature remains neutral with regard to jurisdictional claims in published maps and institutional affiliations. 